\title{
Coupled Mechanical-Electrical-Thermal Modeling for Short-Circuit Prediction in a Lithium-ion Cell under Mechanical Abuse
}

Chao Zhang, Shriram Santhanagopalan, Michael A. Sprague and Ahmad A. Pesaran National Renewable Energy Laboratory, Golden CO 80401

\begin{abstract}
In order to better understand the behavior of Lithium-ion batteries under mechanical abuse, a coupled modeling methodology encompassing the mechanical, thermal and electrical response is presented for predicting short circuit under external crush. The combined mechanical-electric-thermal response is simulated in a commercial finite element software LS-DYNA® using a representative-sandwich finite-element model, where electrical-thermal modeling is conducted after an instantaneous mechanical crush. The model includes an explicit representation of each individual component such as the active material, current collector, separator, etc., and predicts their mechanical deformation under quasi-static compression and indentation. Model predictions show good agreement with experiments: the fracture of the battery structure under an indentation test is accurately predicted. The electrical-thermal simulation predicts the current density and temperature distribution in a reasonable manner. Whereas previously reported models consider the mechanical response exclusively, we use the electrical contact between active materials following the failure of the separator as a criterion for short circuit. These results are used to build a lumped-representative sandwich model that is computationally efficient and captures behavior at the cell level without resolving the individual layers.
\end{abstract}


Keywords: Lithium-ion battery, Short circuit, Multi-physics simulation, Mechanical crush, Representative sandwich model 


\title{
Coupled Mechanical-Electrical-Thermal Modeling for Short-Circuit Prediction in a Lithium-ion Cell under Mechanical Abuse
}

Chao Zhang, Shriram Santhanagopalan, Michael A. Sprague and Ahmad A. Pesaran

\author{
National Renewable Energy Laboratory, Golden CO 80401
}

\section{Introduction}

Lithium-ion batteries are currently the state-of-the-art power sources for a variety of applications, from consumer electronic devices to electric-drive vehicles (EDVs). Being an energized component, failure of the battery is an essential concern, which can result in rupture, smoke, fire, or venting. The failure of Lithium-ion batteries can be due to a number of external abusive conditions (impact/crush, overcharge, thermal ramp, etc.) or internal conditions (internal short circuits, excessive heating due to resistance build-up, etc.), of which the mechanical-abuse-induced short circuit is a very practical problem.

There is a good background in the literature on mechanical properties and mechanical conditions that lead to a short circuit. Mechanical properties of the cells or cell components during intercalation and normal use of batteries are reported extensively in [1-5]. The only publications known to the authors on mechanical properties leading to short circuit in a cell are by Wierzbicki and co-workers [6-9] and by Greve and Fehrenbach [10]. In [6-10], it was observed through experimental measurement of the cell voltage that the instant at which the voltage drop occurs correlates with the instant at which the mechanical failure of the battery structure is observed; macro-scale models were developed and successfully used to predict the fracture of the structure. The short circuit initiation was assumed to be co-located at the same coordinates at which the principal tensile stress first reaches a certain magnitude (for example, $10 \mathrm{MPa}$ in [7]). 
While [6-10] provide a simple and indirect method for predicting a mechanical-abuseinduced short circuit, a coupled mechanical-electrical-thermal (or multi-physics) model that can predict the evolution of short circuit in a straight-forward way, provides more accurate insights into the origin of electrical failure and thermal events that follow.

Multi-physics simulation of Lithium-ion batteries has attracted much attention from researchers [11-15]. However, most of the literatures on multi-physics simulations are restricted to the electrochemical-thermal coupling, or at the individual component level (e.g., only the separator or active-material level) [16-19]. Wu et al. [16] developed a multi-physics model and predicted stress in a separator under intercalation, thermal and mechanical loads. Barai and Mukherjee [19] simulated fracture of electrode active particles due to diffusion-induced stresses using a lattice spring model. Malave et al. [18] identified that surface morphology plays a strong role on the maximum tensile stresses of cathode active particles using a coupled electrochemical-mechanical model. The anisotropic behavior of cathode particles was also simulated and characterized by Malave et al. [18] and Diercks et al. [17]. To the authors' knowledge, the current work is the first publication on coupled mechanical-electrical-thermal simulation of battery failure at the cell level. The next section introduces our approach to coupled modeling and discusses implications for the safety behavior of Lithium-ion cell structure.

\section{Numerical Model}

In this study, a coupled mechanical-electrical-thermal computational model of a pouch battery cell was developed in the commercial finite-element software LS-DYNA [20]. Each individual component of the cell (i.e., active material coating, separator, current collector) was resolved; a representative sandwich finite element model was built, 
which enables the simulation of local damage and short-circuit initiation. A maximum strain failure criterion was applied to the separator layer to represent the mechanical damage to the structure. Subsequently, an electro-thermal simulation is conducted after the mechanical crush, where a criterion for electrical contact is defined between anode and cathode active materials to allow for prediction of the onset of electrical short-circuit.

Each of these steps is described in detail in the next few sub-sections. We start by providing a description of the cell and its mathematical representation. This is followed by a discussion of the material properties and how these are implemented in LS-DYNA. A third sub-section describes coupling of the mechanical models with the wellestablished electrical/thermal model equations. A brief discussion of the results and summary of conclusions is presented at the end.

\subsection{Micro-scale representative sandwich model}

The battery we are studying is a commercially available $740 \mathrm{mAh}$, pouch cell described in [8] comprised of a $\mathrm{LiCoO}_{2}$ cathode and a graphite anode. A schematic of the cell is shown in Fig. 1. The pouch cell was contains two separate laminates; each laminate contains eight repeating units we call a representative sandwich (RS). One RS comprises of one layer of the cathode and anode active materials, two layers of the separator and the two current collectors stacked as shown in Fig. 1. Table 1 lists the number of layers of each cell component in one RS, one battery laminate and the whole pouch cell. As described in Table 1, each pouch cell is comprised of $20 \mathrm{RS}$ units. One simplifying approximation is to represent the $20 \mathrm{RS}$ as one single equivalent but thicker RSs, where each layer has a proportionately larger thickness (20 times the corresponding thickness of each layer). In here we compare two approaches: the first retaining all the 
20 RS units and the second utilizing a single RS with scaled thickness. Description of the numerical implementation is provided next; comparison of the different approaches is presented in Section 3.

Based on symmetry considerations, we consider only one quarter of the in-plane domain in our finite element models (FEM) to save computational time, as shown in Fig. 1(b). The geometry in our FEM has a length $l$ of $25 \mathrm{~mm}$, a width $w$ of $15 \mathrm{~mm}$. The thickness $h$ is $4.6 \mathrm{~mm}$ for a single RS unit. Mechanical damage due to an indentation test, where the cell is subjected to crush by a spherical indenter, as shown in Fig. 1(c) is modeled. The bottom surface of the battery model is constrained by rigid walls. The indentation test is conducted with a rigid sphere moving downward at a speed of $0.4 \mathrm{~m} / \mathrm{s}$. Although the loading speed is much higher than in a typical quasi-static experiment [9], the simulations reported here are still considered quasi-static since we were able to verify that the kinetic energy was less than $1 \%$ of the total energy.

The battery models were meshed using thick shell elements [20], which allow for large aspect ratios and are computationally more efficient than solid elements. Eight nodes were used per element - and one element through the thickness direction was adequate for each component layer. The in-plane element size was selectively refined such that the elements directly under the loading area have an element size of $0.1 \mathrm{~mm}$ and the elements farther out have an element size of $0.7 \mathrm{~mm}$, resulting in a total of 23096 elements for a single RS unit, and 470581 elements for the full pouch cell model. The total computing time using 80 CPUs (Ivy Bridge Xeon processors) is 3 hours for the single RS model and 160 hours for the full pouch cell model.

\subsection{Simulating Mechanical Deformation}


The FEM software package used in our simulations, LS-DYNA, has built-in templates to simulate the mechanical response of a wide range of materials - which can then be adopted for the individual case-study of interest by incorporating suitable material properties.

\subsubsection{Material Properties}

In previous work by Sahraei et al. [8] and Lai et al. [21], a series of experiments were conducted to characterize the tensile and compressive properties of the battery components. It was suggested that the current collectors can be modeled using the piecewise plasticity representation in LS-DYNA. In this work, the MAT_PLASTIC KINEMATIC representation [20], a cost-effective model for isotropic-hardening plasticity, is used to describe the mechanical behavior of the current collectors. The associated properties to be specified as input parameters with this representation in LSDYNA are shown in Table 2. These material properties for the current collectors were obtained by Hill [22] using similar materials.

The mechanical response of the active material layers and the separator are more complicated due to the presence of porosity. We propose an alternate analytical procedure is proposed to estimate the effective compressive stress-strain response of these porous layers from existing compression data experimentally measured using the full pouch cell. Figure 2(a) presents the compressive stress-strain curve of a pouch cell without the casing material [8]. As we can see, the curve can be divided into two stages: a gradual stiffening stage from global strain level $0 \%$ to around $3 \%$ and a linear increasing stage beyond that. Similar trends are reported by Lai et al [21]. The initial gradual stiffening stage is due to the decrease of porosity due to the compressive loading and the 
later linear stage corresponds to the compression response of the compacted layers. The compressive stress-strain responses of individual components (cathode, anode and separator) reported by Sahraei et al. [8] are also consistent with this observation. Based on the two stage responses, a constitutive model is developed to describe the compressive response of the separator and active materials, assuming that the Young's modulus $E$ of the stiffening stage varies exponentially with the strain $\varepsilon$ from initial modulus $E_{0}$ to a fully compacted value $E_{\max }$.

$$
\begin{gathered}
E=\left\{\begin{array}{cc}
E_{\max } e^{\beta\left(\varepsilon-\varepsilon_{p}\right)} & \varepsilon<\varepsilon_{p} \\
E_{\max } & \varepsilon \geq \varepsilon_{p}
\end{array}\right. \\
E_{0}=E_{\max } e^{-\beta \varepsilon_{p}}
\end{gathered}
$$

In Eqs. (1) and (2), $\varepsilon_{p}$ corresponds to the strain when the material is fully compacted. It can be approximated to the initial porosity. The fitting parameter $\beta$ determines the increasing gradient of Young's modulus against strain in the stiffening stage (See Fig. 2(a)). A higher value of $\beta$ indicates a slower increase of modulus with strain. Since the anode and separator are softer than the cathode, we assume that $\beta_{\text {anode }}=\beta_{\text {separator }}=2 \beta_{\text {cathode }}$. By integrating Eq. 1, we calculate the corresponding stress-strain relationship

$$
\sigma=\int_{0}^{\varepsilon} E d \varepsilon=\left\{\begin{array}{cc}
\frac{E_{\max }\left(e^{\beta \varepsilon}-1\right)}{\beta e^{\beta \varepsilon_{p}}} & \varepsilon<\varepsilon_{p} \\
\frac{E_{\max }\left(1-e^{-\beta \varepsilon_{p}}\right)}{\beta}+E_{\max }\left(\varepsilon-\varepsilon_{p}\right) & \varepsilon \geq \varepsilon_{p}
\end{array}\right.
$$

The porosity of the cathode active material and anode active materials are 0.29 and 0.37 obtained from the manufacture. And the mean porosity of the separator is assumed to be 0.2 estimated based on literatures [23, 24]. Due to the absence of experimental data, we also assume that the compacted modulus $E_{\max }$ of cathode and 
anode active materials are the same, and that $E_{\max }$ for the separator is $150 \mathrm{MPa}$, based on similar data for polymeric materials $[8,21,23]$. In the absence of body forces, we also assumed in this work that under a uniform compression test, the effective throughthickness stress $\left(\sigma_{e f f}\right)$ of each layer of the battery cell is the same, as expressed in the equation below:

$$
\sigma_{\text {eff }}=\sigma_{\text {actives }}=\sigma_{\text {collectors }}=\sigma_{\text {separator }}
$$

In order to estimate the stress-strain response of active materials and separator, $\beta_{\text {cathode }}$ and $E_{\max }$ of active materials are the only two unknown parameters to be determined these were regressed based on the iso-stress principle. The effective through-thickness strain $\left(\varepsilon_{e f f}\right)$ can be taken as the volume (thickness) average of strain in each individual component:

$$
\varepsilon_{\text {eff }}=v_{\text {actives }} \varepsilon_{\text {actives }}+v_{\text {collectors }} \varepsilon_{\text {collectors }}+v_{\text {separator }} \varepsilon_{\text {separator }}
$$

where $v_{\text {actives }}, v_{\text {collectors }}$ and $v_{\text {separator }}$ correspond to the ratio of the volume of the respective component to the total volume of the pouch cell. Using known stress-strain responses for the current collectors (Table 2) and the two-stage compressive stress-strain response of the full pouch cell (Fig. 2(a)) together with Eqs. (4) and (5), the value of parameter $\beta_{\text {cathode }}$ is regressed to be 2.1 and $E_{\max }$ of the active materials is $330 \mathrm{MPa}$. As shown in Fig. 2(a), for these parameter values, the experimentally measured compression curve matches well with the analytically predicted curve using Eqs. (3-5).

Once all the parameters have been determined as described above, we then can derive the stress-strain response of the active materials and separator using Eq. (3). These are plotted in Fig. 2(b) - it must be pointed out that it is difficult to obtain such response using experimental compression tests for the porous thin-films - and that we 
report the calculation procedure and results for battery materials here for the first time. Although the approach described above to obtain the constitutive properties for the individual layers adequately represents the full cell response, we reiterate that several simplifying assumptions were made during the derivation of constitutive parameters for the active material layers and the separator due to the lack of experimental data. To develop more accurate constitutive models for the porous active and separator layers, by relaxing some (or all) of the assumptions described earlier in this sub-section, there is a strong need to characterize the compressive mechanical performance of these porous layers. Since the main goal of this work is to simulate the electrical and thermal response, following a mechanical crush, the estimates for the mechanical properties provide adequate basis for our results. Upon availability of experimental data from different test conditions, the constitutive relationship can be suitably updated, without necessitating changes to the other parts of the model equations.

\subsubsection{Mechanical Model}

The compressive stress-strain response of active materials and separators were then implemented into LS-DYNA using the MAT_CRUSHABLE_FOAM material model, which is suitable for modeling deformation of battery materials, with optional damping and fully elastic unloading. We used a damping factor 0.05 to promote numerical stability. Table 3 summarizes the material parameters provided as input to the LS-DYNA model for active materials and the separator. The tensile Young's modulus and tensile cut-off stress (yield stress) are from Lai et al [21]'s measurements for similar materials; the Poisson's ratios are assumed to be zero since no lateral deformation is 
observed for these layers under compression [8]. The active material layers have nearly no tensile resistance, with a tensile cut-off stress as low as $10 \mathrm{MPa}$.

The structural evolution of the cell is analyzed using the LS-DYNA explicit structure solver to obtain the deformed geometry due to mechanical crush. The explicit mechanical solver seeks a solution to the momentum conservation equation:

$$
\sigma_{i j, j}+\rho f_{i}=\rho u_{i, t t}
$$

where $\sigma_{i j}$ denotes components of stress, $u_{i}$ denotes components of stress, $\rho$ is the density, $f_{i}$ is the body force density and $t$ is time. The subscript notation on $\sigma_{i j, j}$ denotes covariant differentiation, similarly, $u_{i, t t}$ represents acceleration. The constituent (stress-strain) relationship (see Eq. (3), for example) can be rewritten as

$$
\sigma_{i j}=C_{i j k l} \varepsilon_{k l}
$$

where $C_{i j k l}$ is the stiffness matrix, and the components of strain $\varepsilon_{k l}$ is related to the displacement by the following relationship:

$$
\varepsilon_{k l}=\frac{1}{2}\left(\frac{\partial u_{k}}{\partial x_{l}}+\frac{\partial u_{l}}{\partial x_{k}}\right)
$$

where $x$ is the geometrical coordinate. The indexes $i, j, k$ and $l$ equal 1,2 , and 3 corresponding to $x, y$ and $z$ directions. A detailed description of the constituent material models we are using is provided in [20].

Corresponding to the test condition shown in Fig. 1(c), the boundary condition at $z=0$ is set by the fixed wall constraint:

$$
\left.\frac{\partial u_{3}}{\partial x_{3}}\right|_{z=0}=0
$$


At $z=h$, for the region directly in contact with the spherical indenter, the $z$-velocity equals that of the indenter, owing to the no-slip condition:

$$
\left.\frac{\partial u_{3}}{\partial x_{3}}\right|_{z=h, \sqrt{x^{2}+y^{2}} \leq r(t)}=0.4 \mathrm{~m} / \mathrm{s}
$$

where $r(t)$ equals the radius of the cross-section of the indenter at $z=h$ for any given instant of time. No constraints are imposed on the strain along all other free edges (i.e., $\left.\sigma_{i j}=0\right)$. Table 4 provides a summary of the boundary conditions.

\subsubsection{Mechanical Failure Criterion}

Simulation of mechanical failure of cell components has rarely been reported in the literature. To simulate mechanical failure, one must consider deletion of elements upon attaining a pre-defined failure criterion, such as a set stress or strain value, for example. Material erosion subject to user-specified criteria is a feature available [20] in LS-DYNA. In this work, based on the constitutive properties chosen for our simulations, we anticipate that the failure of the separator material will occur well in advance before other forms of short-circuit (e.g., anode/aluminum etc.) can take place. Correspondingly, a maximum strain failure criterion was applied for the separator:

$$
\varepsilon \geq \varepsilon_{\max }
$$

We carried out a parametric study on the maximum through-thickness strain value $\left(\varepsilon_{\max }\right)$ of the separators using the single RS model, and found that when $\varepsilon_{\max }=0.93$ (which means that the thickness of the element is compressed to $7 \%$ of initial thickness), the numerically predicted and the experimentally measured global failure strains match (See Fig. 2(d)). Once the failure criterion is satisfied, the element will be deleted from the calculation. The generation of negative volumes is prevented by using a stiffness factor of 
10 for elements with thicknesses below $10 \%$ of the original values, during the indentation simulations.

\subsection{Coupling Mechanical Response with Electrical/Thermal Models}

For the indentation test simulated here, the time scales for the mechanical response are several orders of magnitude smaller compared to that of the electrical and thermal response. We first obtain the stress vs. displacement response through the mechanical solver, the displacements (i.e., the deformed geometry) is then transferred to the electromagnetic (EM) and thermal solvers in LS-DYNA. The present modeling workflow follows a one-way coupling approach, and no information from the electrical or thermal simulations is returned back to the mechanical solver. The limitation of this approach is that we inherently assume that the mechanical deformation resulting from changes to the material properties (e.g., melting of different cell components) is not captured in modeling the thermal/electrical propagation. However, for analysis of the mechanism or origin of short-circuit, the timescales of interest are sufficiently small, as shown in our results and discussion, that this assumption is reasonable. Figure 3 shows a flowchart of the mechanical-electrical-thermal coupled modeling approach. Electricalthermal simulations are performed on the deformed geometry at various stages of the crush process.

\subsubsection{Electrical Model}

The electrical simulation uses a resistive heating model [20]. The model equations correspond to the electrochemical model developed by Fuller et al. [26] where charge transport is dominated by electronic conductivity across the short-circuit. The main equations for the resistive heating model are listed below: 


$$
\begin{gathered}
\vec{j}=-\kappa_{s} \vec{\nabla} \varphi \\
\vec{E}=-\vec{\nabla} \varphi \\
\mathrm{E}_{\text {Joule }}=\frac{\vec{j} \cdot \vec{j}}{\kappa_{s}}
\end{gathered}
$$

where $\vec{j}$ is the current density, $\kappa_{s}$ corresponds to electronic conductivity across the different solid phases, $\varphi$ is electrical potential, $\vec{E}$ the electrical field and $\mathrm{E}_{\text {Joule }}$ indicates the Joule heating energy associated with the electronic flow. Over the duration of a few seconds, ionic diffusion and contributions from the electrolyte to the short-circuit are assumed to be negligible - given that the difference between the electronic and ionic conductivities are four orders in magnitude or larger.

\subsubsection{Thermal Model}

For the thermal solver, the governing equation for conduction of heat in a threedimensional solid is given by [27]:

$$
\rho c \frac{\partial T}{\partial t}=\frac{\partial}{\partial x_{i}}\left[K_{i j} \frac{\partial T}{\partial x_{j}}\right]+Q
$$

In Eq. (15), $T$ is temperature, $c$ is specific heat, $K_{i j}$ is thermal conductivity and $Q$ indicates heat generation rate per unit volume. In this work, the joule heating is the only source of heat:

$$
Q=\frac{\dot{\mathrm{E}}_{\text {Joule }}}{V}
$$

where the dot indicates time derivative, and $V$ is the volume of the element.

The default adiabatic boundary condition is used on all free surfaces. Energy is 
conserved along the contact surfaces. Table 4 lists the boundary conditions and Table 5 shows the electrical conductivity and thermal properties used in our electrical-thermal analyses.

Both the thermal and the EM model equations are solved implicitly. At each EM/thermal time step, the EM model will output the Joule heating power term (Eq. (14)), which the thermal solver will utilize to compute the temperature $T$, as shown in Fig. 3. In this work, due to the lack the experimental data, the electrical conductivities of the battery components are assumed to be independent with temperature (i.e., constant over time). However, there are several options available in LS-DYNA that permit the user to define electrical conductivity as a function of temperature.

Periodic snapshots of the deformed geometry from the mechanical simulations are exported to the electrical/thermal analysis. A total of 12 (one for every $5 \%$ global strain) deformed geometries were exported to predict the corresponding electrical and thermal response during the mechanical crush. The cell is at a fully charged state at the beginning of the simulation - the cell voltage is at $4.2 \mathrm{~V}$ between the two current collectors, and an increase in current density is expected when the short circuit initiates. The evolution of voltage after initiation of a short circuit is monitored through calculation of the voltage drop caused by the decrease in resistance due to the short-circuit. Table 5 summaries the boundary conditions for the contact interfaces before and after mechanical failure of the separator. For the interfaces between different layers, continuity boundary conditions are applied if there is no element failure between them, or the contact boundary conditions listed in the table will be applied.

2.3.3 Failure Criteria for the Electrical/Thermal Models 
Mechanical failure across an element does not automatically result in an electrical short-circuit across the different cell components. This is a crucial distinction to make, when resolving the electrical resistance across the short-circuit. Whereas existing models assume initiation of electrical contact following mechanical failure of a given cell component, our approach calculates the current flow across the component layers that border the element subjected to mechanical failure and utilizes the resultant voltage drop to determine the short resistance. This process allows us to introduce additional failure criteria based on the electrical and thermal properties of the different layers coming into contact across the instance of mechanical failure. For instance, in this work, the criterion for electrical failure is the instantaneous local current density exceeding a preset value, for example, $0.01 \mathrm{C}$, which corresponds to a current density of $13.12 \mathrm{~mA} / \mathrm{mm}^{2}$ for a 740 $\mathrm{mAh}$ cell when the area of elements subject to mechanical failure is $0.785 \mathrm{~mm}^{2}$. Thermal failure is set to initiate when the temperature across the element in question exceeds the melting point of the corresponding material (e.g., $144{ }^{\circ} \mathrm{C}$ or $417 \mathrm{~K}$ for the separator). However, mechanical failure sets in instantaneously compared to the thermal response, which is usually distributed over a span of seconds. Accordingly, in this work, we present our methodology for linking the mechanical-failure criterion with the follow-on electrical activity and discuss some preliminary thermal responses. Simulation of the propagation of the electrochemical and thermal behavior of the cell across a timescale of several seconds is not within our intended scope for the present study. However, interested enduser can incorporate additional heat source terms by replacing $Q$ in Eq. (12) with a summation of the heat generated from multiple sources, such as chemical reactions, ionic resistivity, etc., as appropriate for the timescale of interest. 


\section{Results and Discussion}

\subsection{Calibration of the mechanical model}

The mechanical model is validated through comparison of the numerically predicted and experimentally measured force vs. global-strain curves (See Fig. 2c). Utilizing the constitutive relationships shown on Fig. 2b, the mechanical response of the cell subjected to indentation using a spherical indenter of $12.7 \mathrm{~mm}$ diameter was simulated. Experimental indentation tests corresponding to these results, on the pouch cell described in Section 2 were conducted by Sahraei et al. [8] using a hemispherical punch at a rate lower than $3 \mathrm{~mm} /$ minute and monitoring the voltage simultaneously. A significant finding of the experimental results was the fact that the electrical short circuit detected from the drop in cell voltage coincided well with the drop in the load due to mechanical failure. No temperature data is available for this test.

In our work, the global strain is defined as the applied displacement of rigid sphere over the initial thickness of the model. The force is recorded as the reaction force of rigid sphere in the through-thickness direction (z-direction). Figure 2(c) presents a comparison of the simulated and experimental results. Both the full pouch-cell model and the single RS model predict well the force-strain responses under indentation, showing the suitability of the materials models presented here to capture the mechanical response of the pouch cell components, and the viability of using the single RS model to predict the global mechanical response of the full pouch-cell. The latter observation is important when scaling these models to simulate the response of multi-cell modules or battery packs. The single-RS model is capable of predicting the onset of failure within the pouch 
cell under indentation - and enables a lumped representation of the cell, with the ability to incorporate the mechanical properties of the individual cell components.

There are some notable differences between the single RS and the full pouch-cell models. For instance, on Fig. 2c, the full pouch-cell model does not show any abrupt changes to the force, whereas the single-RS model predicts a sudden drop in the force after $8 \mathrm{kN}$, corresponding to the failure of the separator layers. This is attributed to the fact that on the full pouch-cell model, the individual layers are very thin and as a result, the failure of the separator layers may not affect much the mechanical resistance of the structure, whereas, in the RS representation all the separator layers are lumped together, and there is an implicit assumption that all the layers fail at the same time. Thus, the RS model is better suited to identify the onset of the short-circuit, but, depending on the loading condition, the results for propagation of failure may differ from the experimental observations: for instance, the RS model is adequate to simulate the impact response where an instantaneous load sufficient to mechanically damage the cell is applied. However, it cannot adequately distinguish the differences between the mechanical response of two cell designs, one with thin electrodes, versus the other with thicker but fewer electrodes, if the total thickness of all the electrode layers in question remains the same. It is pertinent to conduct systematic experimental and numerical studies to investigate the damage evolution process of the multilayered cell structure under indentation.

\subsection{Calibration of the Failure Strain}

Based on the parameter values shown in Tables 2 and 3, for our case study, the separator is the first component to undergo mechanical failure. In this work, we present 
our analyses based on this parameter set, although this result must be evaluated on a caseby-case basis, for different cell components with variations in mechanical properties and cell design. Figure 2(d) shows the parametric correlation of separator failure strain $\varepsilon_{\max }$ through comparing the single-RS modeling and experimental results. As we can see, with the increase of $\varepsilon_{\max }$, the global failure strain and applied force on the battery structure increases. The numerical value for the predicted global failure strain matches with the experimental result shown on Fig. 2(c), when $\varepsilon_{\max }=0.93$.

Differences in the failure strain $\left(\varepsilon_{\max }\right)$ of the separator will also result in different contact areas at the onset of mechanical failure, because more elements will meet the failure criteria when the failure strain is larger. And the contact area has a significant impact on the short-circuit behavior. Figure 4 compares the deformed geometry after onset of failure as predicted numerically from the RS model and element erosion in the separator layer for three different failure strain values: $0.8,0.93$ and 0.96 . The deformation contours of the geometry show the through-thickness displacement, from which we can see that the maximum displacement of the indenter from $z=0$ increases by $24.1 \%$ when the failure strain increases from 0.8 to 0.93 and $13.3 \%$ when the failure strain increases from 0.93 to 0.96 . The eroded volume fraction at the moment of structural failure also increases proportionately, with the failure strain. Under identical conditions, a larger eroded volume fraction indicates a larger electrical contact area between the cathode and anode active layers, which will produce a more significant short (lower resistance values, larger current and faster voltage drop). The impact of contact area on the short-circuit behavior will be discussed in a later section along with the thermal and electrical response. 


\subsection{Evolution of Mechanical Damage}

Another advantage of the single RS model is its capability in predicting the effective response of each individual component and thus, providing for an easier interpretation of the data. The maximum through-thickness strain for each component under indentation, which occur at the center of the plate under indenter (i.e., $x=y=0$ and $z=h$ on Fig. 1(b)), are shown in Fig. 5(a). As we can see in Fig. 5(a), the separator undergoes much larger deformation compared with the other components due to its much lower stiffness. A second observation is that the top of the structure (i.e., $z=h$ ) deforms the most due to the localized loading condition for the indenter. The current collectors experience almost negligible deformations. Figure 5(b) shows the contours of the through-thickness strain for the single-RS model at different global strain levels, which provides insights on the damage evolution within the structure. At the initial stage, the top layers, especially the top separator layer (Layer 1 on Fig. 5(b)), suffer more deformation (i.e., higher displacements) due to the localized loading. As the indenter goes down further, the separator at the middle of the structure (Layer 5) starts deforming and displays higher strain than the top anode layer (Layer 2) at a global strain level of $44 \%$. When the global strain level reaches $62 \%$, the criterion for mechanical failure (Eq. 11) is satisfied and element deletion occurs at the mid separator layer (Layer 5). The predicted values for strain and deformation for each cell component under indentation are in line with their constitutive properties (Fig. 2(b)). Following the failure of separators, as shown in Fig. 5, the cathode active and anode active layers are compressed and come into electrical contact. A short circuit initiates when the criterion for electrical contact (See 
Section 2.3.3) is satisfied, between the cathode active layer (Layer 6) and the anode active layer (Layer 4) next to the separator.

\subsection{Electrical response and short-circuit prediction}

Next, we present results of electrical simulations on the deformed RS model. In our case, the cell is at a $100 \%$ state of charge, and a constant voltage (4.2V) is applied between the cathode and anode current collectors (Layers 2 and 8 on Fig. 5(b)), and an electrical short that initiates following the failure of the separator layer (5th layer as shown in Fig. 5(b)). Before we discuss the results in detail, it must be pointed out that the single RS model does not provide information on the specific location where the electrical short-circuit initializes. For instance, the single RS model does not specify which two layers of active material on the full pouch-cell geometry shown on Fig. 1(a) initiate electrical contact. In a realistic case, for a full pouch-format cell under indentation, the short circuit should occur at the top few layers where mechanical damage sets in first. The exact location of short circuit can be predicted accurately by using the full pouch cell model, utilizing the same approach discussed here; however, the effort will require significantly larger computational time.

The effective electrical resistance $(R)$ of a homogenous material follows the following equation:

$$
R=\frac{L}{A \kappa_{s}}=\frac{L_{0}\left(1-\varepsilon_{z}\right)}{A \kappa_{s}}=R_{0}\left(1-\varepsilon_{z}\right)
$$

where $A$ is in-plane area, $L$ and $L_{0}$ denote thickness as a function of time and the initial thickness respectively, $R_{0}$ is the initial resistance, and $\varepsilon_{z}$ is the through-thickness strain. Due to the porous nature of the active material layers and the separator, there will be very minor variation in the in-plane (i.e., $x-y$ plane) dimensions. As such, the effective 
resistance of each component will have a nearly linear relationship with its local throughthickness strain. The element at the center of each layer (i.e., $x=y=0$ ), which has maximum through-thickness strain histories of each component under indentation (See Fig. 5(a)), will have the most significant change in resistance. Using Eq. (17), we can determine the evolution of the resistance $\left(R / R_{0}\right)$ of each component under indentation, before initiation of electrical contact, as shown in Fig. 6(a). The third to seventh layers of the single RS are selected to study the electric responses of each component.

Since there is a very minor change in voltage before the short, we assume a constant voltage before the occurrence of the short circuit. The electronic current density (j) across each layer will then follow relationship shown below:

$$
j=\frac{V}{R}=\frac{V A \kappa_{s}}{L}=\frac{V A \kappa_{s}}{L_{0}\left(1-\varepsilon_{z}\right)}=\frac{j_{0}}{\left(1-\varepsilon_{z}\right)}
$$

where $V$ is the applied voltage, and $j_{0}$ is the corresponding initial current density across each of the component layers. From Eq. (18), we find that the current density varies inversely with the effective resistance $(R)$ and the through-thickness strain. Figure 6(b) compares the history of the normalized current density across each component against the global strain. The normalized current density before the initiation of an electrical contact across the separator and active materials all increase with an increase in time, due to the decreasing thickness. Note that $j_{0}$ is calculated using the respective electronic conductivity values for the different cell components (See Table 4) and thus, the numerical values for $j$ and $j_{0}$ for the separator will be very small, compared to that across the current collectors for the same voltage drop. Thus, although the normalized current density across the separator increases exponentially with time (or global strain), the magnitude of $j$ across the separator, is still much lower than that for the other components, 
since the electronic conductivity of the separator is assumed to be over six orders of magnitude smaller than that of the other layers. This indicates that there is little chance of electrical contact due to an ohmic drop in the cell voltage resulting from just a reduction in the thickness of the components. Under these circumstances, in the absence of thermal failure, the most likely origin of short circuit will be the mechanical failure of one or more layers. This result also highlights the importance of good mechanical failure strain for the separator, which has a significant influence on the facture strain of the cell structure under the conditions presented here.

The electrical response of the cell after initiation of short circuit is discussed next. Figure 7(a) shows snapshots of the current density across the cathode active layer (Layer 6 on Fig. 5(b)) and contours of current density at different stages during the indentation test. There is a small increase in the current density before the onset of failure, due to the decrease in thickness; but a sharp increase in current density is detected at the moment of failure due to the initiation of short circuit, which corresponds to the contour plot shown on Fig. 7(a), for the global strain level of $62 \%$. As shown on the current density vector plots, initially for the undeformed geometry the current flow between the layers is small, and occurs in a direction normal to the surface and has the same small magnitude across the different layers, due to the poor electronic conductivity of the separator. Following a deformation, the current density is highly concentrated at the location of mechanical damage and the current density vectors display an evolution in the direction of the flux with the mechanical deformation of the geometry. At the instant of short-circuit, the current flows across each of the adjacent active material layers in the in-plane direction, towards the region of electrical contact (which in this case is the location of separator 
failure), through-plane across the layers at the location of short-circuit, and then from there to the subsequent layers. Note the logarithmic scale on the ordinate of Fig. 7(a) indicating the sudden surge in the current density by several orders of magnitude, following the short-circuit.

With the initiation of electrical contact, the voltage and resistance of the cell structure drops to very small values. Understanding the voltage evolution behavior after the short circuit is of great significance, both for designing a safer battery, as well as for design of post-event containment measures. Considering only the through-thickness cross-section of the structure (1D space), the voltage-drop across the cell, calculated after short-circuit can be considered as the summarization of voltage drop across each individual layer. Before short, due to the deformation induced decreasing of resistance, there will be a very small drop in voltage. Compared with the instantaneous voltage drop after the short, the voltage change before short-circuit is negligible. As the short progresses, there is a decrease of current density, resulting in a decrease of the rate of change in the cell voltage. Figure $7(\mathrm{~b})$ shows the evolution of voltage and contact area during the indentation test. The contact area history is determined as the summation of inplane area of elements that have met the failure criteria outlined in Section 3.3, at each instant. Thus, before short, the contact area is zero and starts increasing continuously with the failure of the separate layer between cathode and anode. As we can see from Fig. 7(b), the voltage drops instantaneously against the strain increment. There is little change to the cell voltage before short-circuit. The voltage drop, and correspondingly, the change in the short circuit area start-off with a step change, but quickly level off with the evolution of the short. Notice also that the voltage drop occurs over a range of 0.64 to 
$0.67 \mathrm{~mm} / \mathrm{mm}$ whereas the evolution of the contact area is a much slower process. However, as shown in the inset of Fig. 7(b), there is a gradual decrease in the voltage drop with the evolution of the short-circuit. Recently, Ramadass et al. [28] and Fang et al. [29] presented experimental and modeling results simulating the internal short behavior in a Li-ion cell under nail penetration. According to their results [28], the voltage is almost constant before the occurring of a short and there is a gradual decrease of voltage after short taking about two seconds for a $6.28 \mathrm{~mm}^{2}$ circular electric contact area.

As mentioned in the previous section, the electric contact area has a significant impact on the short-circuit behavior. To elucidate the effect of contact area, the electrical response of the deformed geometry (Fig. 4) for three different values of the contact area are compared next. The contact areas of deformed RS model with failure strain $0.8,0.93$ and 0.96 are $2.775,4.125$ and $6.15 \mathrm{~mm}^{2}$, respectively. The current density distribution contours and flow directions of these three models at the moment of short are similar to Fig. 7(a) but with different magnitudes, increasing from 568.9 to 699.5 and then 1319 $\mathrm{A} / \mathrm{mm}^{2}$ with the increase in contact area. This is due to the relatively lower short resistance when there is a larger electric contact area. A higher current density will result in a faster voltage drop, as shown in Figure 8(a). For the same cathode-anode short, the voltage drop from $4.2 \mathrm{~V}$ to $100 \mathrm{mV}$ for an electrical contact area of $1.53 \mathrm{~mm}^{2}$ is 4 times faster than that for $0.694 \mathrm{~mm}^{2}$. This result, when extrapolated, is useful in explaining why short-circuits involving very small contact areas do not result in thermal runaway - the voltage drop is so slow that any heat generated from such shorts is dissipated before sufficient temperature rise can result in a runaway. On the other hand, when the contact area is exceedingly large, the energy content of the small format cell is released within 
milliseconds before the current density can rise to sufficiently large values to generate any heat; whereas, a large-format cell will continue to have sufficient energy available across the same time-scale, and will generate sufficient heat to result in the follow-on reactions. The short resistance is also associated with the conductivity of materials involved in electric contact. As discussed by Fang et al [29], the short resistance of anode-cathode short is much higher than anode-aluminum short. Using arguments similar to what we just presented, the presented model can be used to study different short conditions resulting from contact across different layers.

\subsection{Thermal response}

In the previous section, we demonstrated the capability of our model in predicting the mechanical and electric response of a battery cell during mechanical crush. The thermal response is of critical interest in understanding the safety behavior and its potential impact on propagation of failure. Towards this end, we solve the energy balance equation on the deformed geometry to obtain the rise in the local temperature across different locations of the geometry subjected to crush. Figure 8(b) shows the maximumtemperature history and temperature contour plots for selected stages of the cell after the occurrence of the short. It is observed that the temperature increases gradually and the maximum increase in the local temperature was found to be about $22.75 \mathrm{~K}$. Also, in our simulations, there is no instantaneous rise in temperature observed upon incurring an internal short, similar to the experimentally observed results reported by Ramadass et al. [28]. The initial rise in temperature corresponds to the fastest rate at which the potential across the layers drops. The local temperature then levels off and subsequently starts decreasing due to the dissipation of the heat via conduction across the electrode layers. 
Note that the time scale for our simulations is less than 2 seconds - within this short time frame, the temperature rise is mainly from the joule heating of the different layers across the short-circuit. In this case, the temperature rise was not sufficient to trigger follow-on reactions. However, for a short with higher resistance, the temperature rise will likely be higher. These results with contribution to the heat source from different thermal runaway reactions across multiple time constants, were discussed in an earlier work [30]. Also shown in Figure 8(b), is the history of the maximum temperature during short circuit for different contact areas. The location of maximum local temperature coincides with the location of the maximum local resistance - which in this case is the separator element closest to the short-circuit area. As we can see, the temperature starts decreasing earlier for the short with larger contact area due to the faster voltage drop. Also, the maximum temperature for a larger value of contact area is lower due to quick energy release.

\section{Conclusions}

In this work, we presented a mechanical-electrical-thermal modeling approach for predicting mechanical abuse induced short circuit behavior of lithium ion cells. A finite element representative sandwich model was developed and solved using the explicit mechanical solver and electric-thermal solver in LS-DYNA in a sequential way. The model results correlate well with experiments in predicting the mechanical response, successfully predict the initiation of short circuit due to structural fracture and post-short thermal and electric responses. From the instance of short-circuit, the magnitude of current density increases about $10^{5}$ times. It is found that there is a gradual decreasing in the voltage drop with the evolution of the short-circuit. No instantaneous rise in temperature was observed upon incurring internal short. Instead, the cell temperatures 
increased gradually to a peak value and then start decreasing. The effect of contact area at the moment of short was investigated. It is found that a larger contact area will result in a faster voltage drop and a lower local maximum temperature.

The modeling technique is useful in studying the safety behavior of Lithium-ion cells under mechanical abuse and can be helpful in the design of more efficient and safer battery structures. Future work should focus on obtaining relevant constitutive properties for the cell components subjected to mechanical crush, measurement of temperature and voltage profiles under these test conditions. The subsequent correlation of electrical and thermal responses with the prediction of electrochemical-thermal behavior after shortcircuit initiation is also of tremendous interests to the battery community.

\section{Acknowledgment}

This study was supported by the Vehicle Technologies Office, Office of Energy Efficiency and Renewable Energy, U.S. Department of Energy. The authors would like to thank Dr. Elham Sahraei Esfahani and Prof. Thomasz Wierzbicki at the Impact and Crashworthiness Laboratory, Massachusetts Institute of Technology, for their insights and discussion of the data presented in their earlier work [8-9]. Access to high performance computing (HPC) resources at National Renewable Energy Laboratory is also gratefully acknowledged. 


\section{References}

[1] S. Golmon, K. Maute, M.L. Dunn, Comput. Struct. 87 (2009) 1567-1579.

[2] X. Zhang, W. Shyy, A.M. Sastry, J. Electrochem. Soc. 154 (2007) A910.

[3] X. Zhang, A.M. Sastry, W. Shyy, J. Electrochem. Soc. 155 (2008) A542.

[4] R. Fu, M. Xiao, S. Choe, J. Power Sources 224 (2012) 211-224.

[5] D. Shi, X. Xiao, X. Huang, H. Kia, J. Power Sources 196 (2011) 8129-8139.

[6] T. Wierzbicki, E. Sahraei, J. Power Sources (2013) 467-476.

[7] E. Sahraei, J. Campbell, T. Wierzbicki, J. Power Sources 220 (2012) 360-372.

[8] E. Sahraei, R. Hill, T. Wierzbicki, J. Power Sources 201 (2012) 307-321.

[9] E. Sahraei, J. Meier, T. Wierzbicki, J. Power Sources 247 (2014) 503-516.

[10]L. Greve, C. Fehrenbach, J. Power Sources 214 (2012) 377-385.

[11] X. Xiao, W. Wu, X. Huang, J. Power Sources 195 (2010) 7649-7660.

[12] M. Guo, GH. Kim, R.E. White, J. Power Sources 240 (2013) 80-94.

[13] GH. Kim, K. Smith, KJ. Lee, S. Santhanagopalan, A. Pesaran, J. Electrochem. Soc. 158 (2011) A955-A969.

[14] A.A. Franco, RSC Advances 32(2013) 13027-13058.

[15] JH. Song, SJ. You, DH. Jeon, J. Appl. Electrochem. 44 (2014) 1013-1023.

[16] W. Wu, X. Xiao, X. Huang, S. Yan. Comp. Mater. Sci. 83 (2014) 127-136.

[17] D.R. Diercks, M. Musselman, A. Morgenstern, T. Wilson, M. Kumar, K. Smith, M. Kawase, B.P. Gorman, M. Eberhart, C.E. Packard. J. Electrochem. Soc. 161 (2014) F3039-F3045.

[18] V. Malavé, J.R. Berger, H. Zhu and R.J. Kee. Electrochimica Acta 130 (2014) 707717. 
[19] P. Barai and P.P. Mukherjee. J. Electrochem. Soc. 160 (2013) A955-A967.

[20] J.O. Hallquist. Livermore Software Technology Corporation, 2007.

[21] W.J. Lai, M.Y. Ali and J. Pan. J. Power Sources 245 (2014) 609-623.

[22] R.L. Hill Sr. Doctoral Dissertation Massachusetts Institute of Technology, 2011

[23] J. Cannarella, X.Y. Liu, C.Z. Leng P.D. Sinko, G.Y. Gor and C.B. Arnold. J. Electrochem. Soc. 161 (2014) F3117-F3122.

[24] G.Y. Gor, J. Cannarella, J.H. Prevost and C.B. Arnold. J. Electrochem. Soc. 161 (2014) F3065-F3071.

[25] W.B. Gu and C.Y. Wang. Proc. Electrochem. Soc 99 (2000) 748-762.

[26] T.F. Fuller, M. Doyle and J. Newman. J. Electrochem. Soc. 141 (1994) 1-10.

[27] E.A. Thornton. J. Aircra. 29 (1992) 485-498.

[28] P. Ramadass, W.F. Fang and Z.M. Zhang. J. Power Sources 248 (2014) 769-776.

[29] W.F. Fang, P. Ramadass and Z.M. Zhang. J. Power Sources 248 (2014) 1090-1098.

[30] S. Santhanagopalan, P. Ramadass and J.Z. Zhang, J. Power Sources 194 (2009) 550557. 


\section{TABLES}

Table 1: Summary of the number of layers of each component in the different models

\begin{tabular}{ccccccc}
\hline & Separator & $\begin{array}{c}\text { Cathode } \\
\text { Active }\end{array}$ & $\begin{array}{c}\text { Cathode } \\
\text { Collector }\end{array}$ & $\begin{array}{c}\text { Anode } \\
\text { Active }\end{array}$ & $\begin{array}{c}\text { Anode } \\
\text { Collector }\end{array}$ & $\begin{array}{c}\text { Total } \\
\text { layers }\end{array}$ \\
\hline Single RS & 2 & 2 & 1 & 2 & 1 & 8 \\
$\begin{array}{c}\text { One Battery } \\
\text { Laminate }\end{array}$ & 20 & 20 & 10 & 20 & 11 & 81 \\
Pouch Cell & 41 & 40 & 20 & 40 & 22 & 163 \\
\hline
\end{tabular}

Table 2: Mechanical parameters for current collectors of a Lithium-ion cell [22]

\begin{tabular}{ccccc}
\hline & $\begin{array}{c}\text { Young's Modulus } \\
(\mathrm{GPa})\end{array}$ & $\begin{array}{c}\text { Poisson's } \\
\text { Ratio }\end{array}$ & $\begin{array}{c}\text { Yield Stress } \\
(\mathrm{MPa})\end{array}$ & $\begin{array}{c}\text { Tangent Modulus } \\
(\mathrm{MPa})\end{array}$ \\
\hline $\begin{array}{c}\text { Cathode } \\
\text { Collector } \\
\begin{array}{c}\text { Anode } \\
\text { Collector }\end{array}\end{array}$ & 70 & 0.36 & 180 & 700 \\
\hline
\end{tabular}

Table 3: Mechanical parameters for active materials and separator of the Lithium-ion cell

(See Section 2.2 for details on these parameters).

\begin{tabular}{cccccccc}
\hline & $\begin{array}{c}E_{\max } \\
(\mathrm{MPa})\end{array}$ & Porosity & $\beta$ & $\begin{array}{c}\text { Tensile Young's } \\
\text { Modulus (GPa) }\end{array}$ & $\begin{array}{c}\text { Poisson's } \\
\text { Ratio }\end{array}$ & $\begin{array}{c}\text { Tensile } \\
\text { Cutoff (MPa) }\end{array}$ & $\begin{array}{c}\text { Damping } \\
\text { factor }\end{array}$ \\
\hline $\begin{array}{c}\text { Cathode Active } \\
\text { Material }\end{array}$ & 330 & 0.29 & 2.1 & 5.1 & 0 & 10 & 0.05 \\
$\begin{array}{c}\text { Anode Active } \\
\text { Material }\end{array}$ & 330 & 0.37 & 4.2 & 4.7 & 0 & 10 & 0.05 \\
Separator & 150 & 0.2 & 4.2 & 0.5 & 0 & 100 & 0.05 \\
\hline
\end{tabular}


Table 4: Boundary conditions for the mechanical-EM-thermal solvers. (Where superindex + and - indicates the top and bottom surface, index $k$ indicates the two surfaces been in contact and $n$ is a unit outward normal to the boundary element at the contact interface.)

\begin{tabular}{|c|c|}
\hline & Boundary conditions \\
\hline Mechanical solver & $\begin{array}{l}\text { Free surfaces: } \sigma_{i j}=0 \text { at } \mathrm{x}=-\mathrm{w}, \mathrm{y}=-1 ; \\
\text { Bottom surface constrained: } u_{3}=0 \text { at } \mathrm{z}=0 ; \\
\text { Symmetry surfaces: } u_{2}=0 \text { at } \mathrm{y}=0 ; u_{1}=0 \text { at } \mathrm{x}=0 \text {; } \\
\text { Contact surfaces: }\left(\sigma_{i j}^{+}-\sigma_{i j}^{-}\right) n_{i}=0 \text {. }\end{array}$ \\
\hline EM solver & $\begin{array}{l}\text { Free surfaces: } \nabla \phi=0 \text { for all external surfaces; } \\
\text { Contact surfaces: } \sum_{k=a, b}\left(\kappa_{s}^{k} \frac{\partial \phi_{k}}{\partial n_{k}^{+}}+\kappa_{s}^{k} \frac{\partial \phi_{k}}{\partial n_{k}^{-}}\right)=0\end{array}$ \\
\hline Thermal solver & $\begin{array}{l}\text { Adiabatic: } \frac{\partial T}{\partial n_{i}}=0 \text { for all external surfaces; } \\
\text { Contact surfaces: } \sum_{k=a, b}\left(K_{i j}^{+} \frac{\partial T_{k}}{\partial n_{k}^{+}}+K_{i j}^{-} \frac{\partial T_{k}}{\partial n_{k}^{-}}\right)=0 .\end{array}$ \\
\hline
\end{tabular}


Table 5: Electrical-thermal parameters for each component of the Lithium-ion battery [25].

\begin{tabular}{cccccc}
\hline & Separator & $\begin{array}{c}\text { Cathode } \\
\text { Active }\end{array}$ & $\begin{array}{c}\text { Cathode } \\
\text { Collector }\end{array}$ & $\begin{array}{c}\text { Anode } \\
\text { Active }\end{array}$ & $\begin{array}{c}\text { Anode } \\
\text { Collector }\end{array}$ \\
\hline $\begin{array}{c}\text { Electrical conductivity } \\
(\mathrm{S} / \mathrm{m})\end{array}$ & $1.0 \mathrm{E}-5$ & 38 & $3.8 \mathrm{E}+7$ & 100 & $6.0 \mathrm{E}+7$ \\
$\begin{array}{c}\text { Thermal conductivity } \\
(\mathrm{W} / \mathrm{cm} \bullet \mathrm{K})\end{array}$ & 0.01 & 0.05 & 2.0 & 0.05 & 3.8 \\
Heat capacity $(\mathrm{J} / \mathrm{g} \bullet \mathrm{K})$ & 0.7 & 0.7 & 0.870 & 0.7 & 0.381 \\
\hline
\end{tabular}




\section{FIGURES}

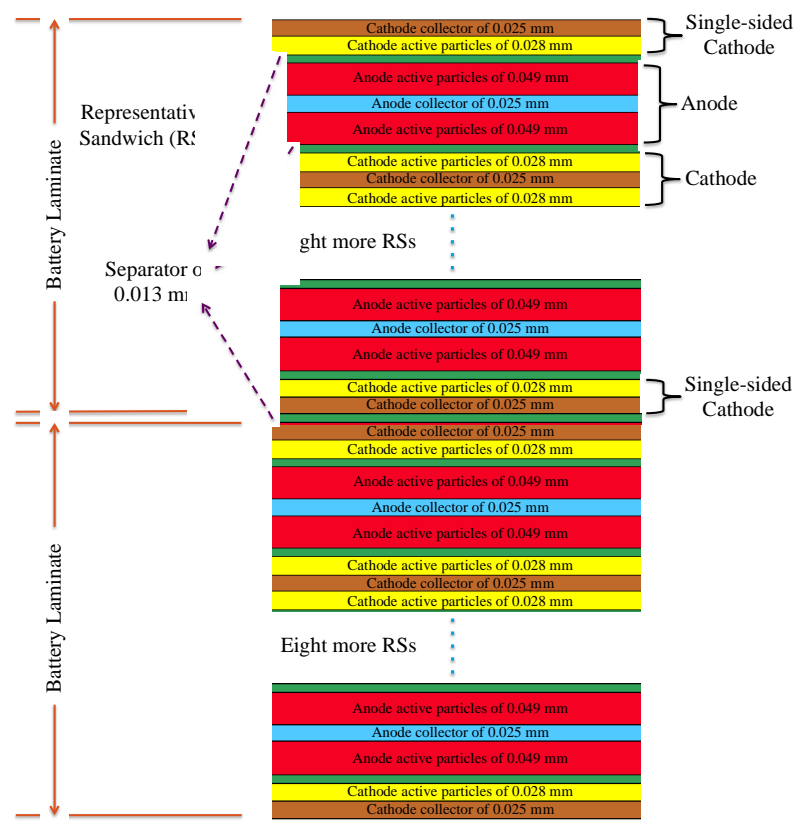

(a)

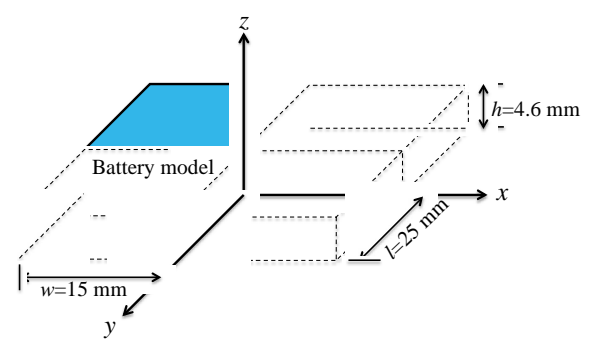

(b)

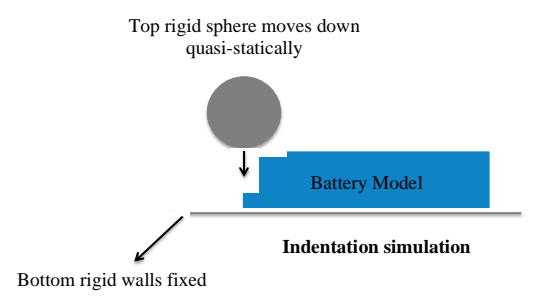

(c)

Figure 1 


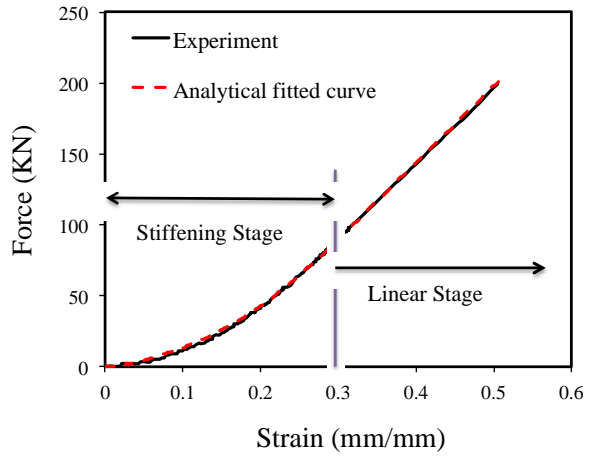

(a)

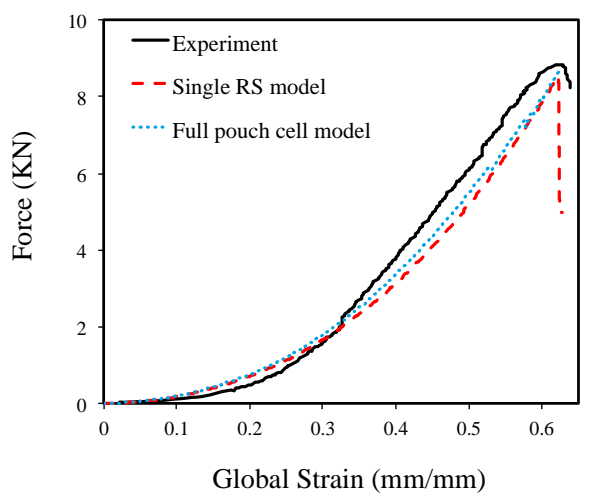

(c)

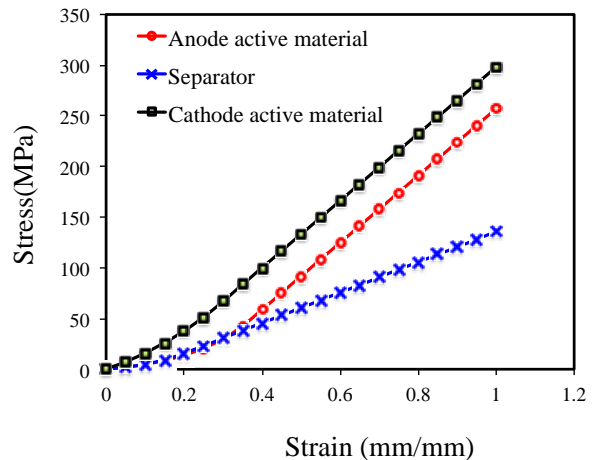

(b)

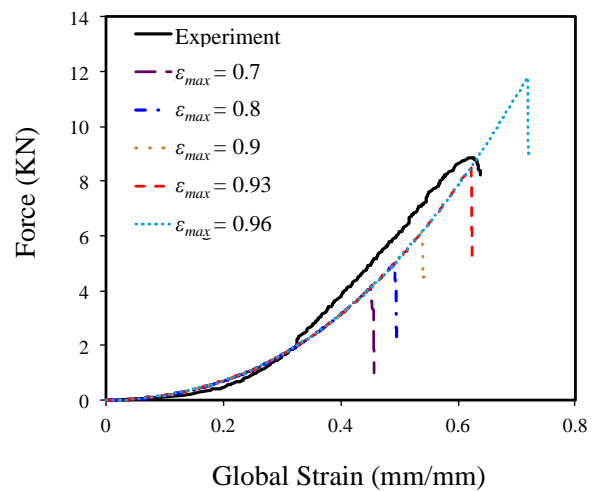

(d)

Figure 2 


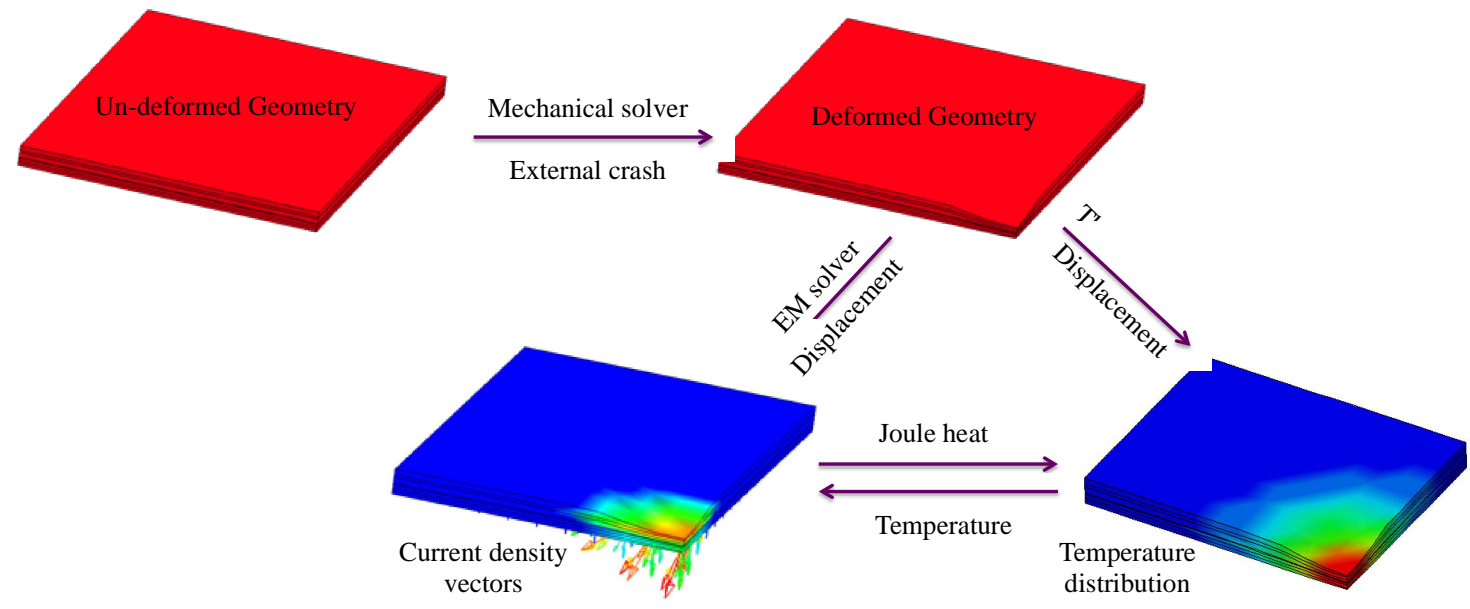

Figure 3 


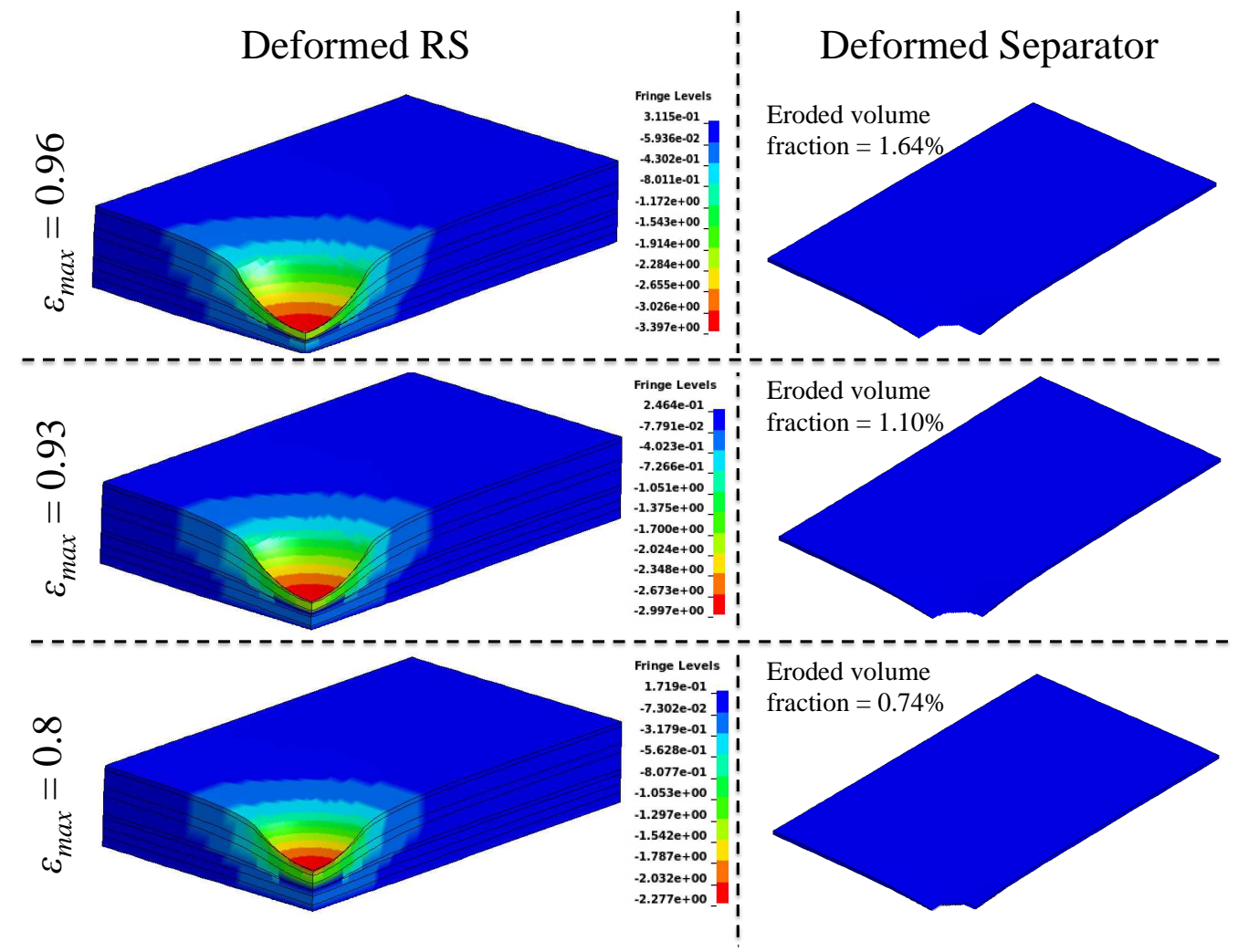

Figure 4 


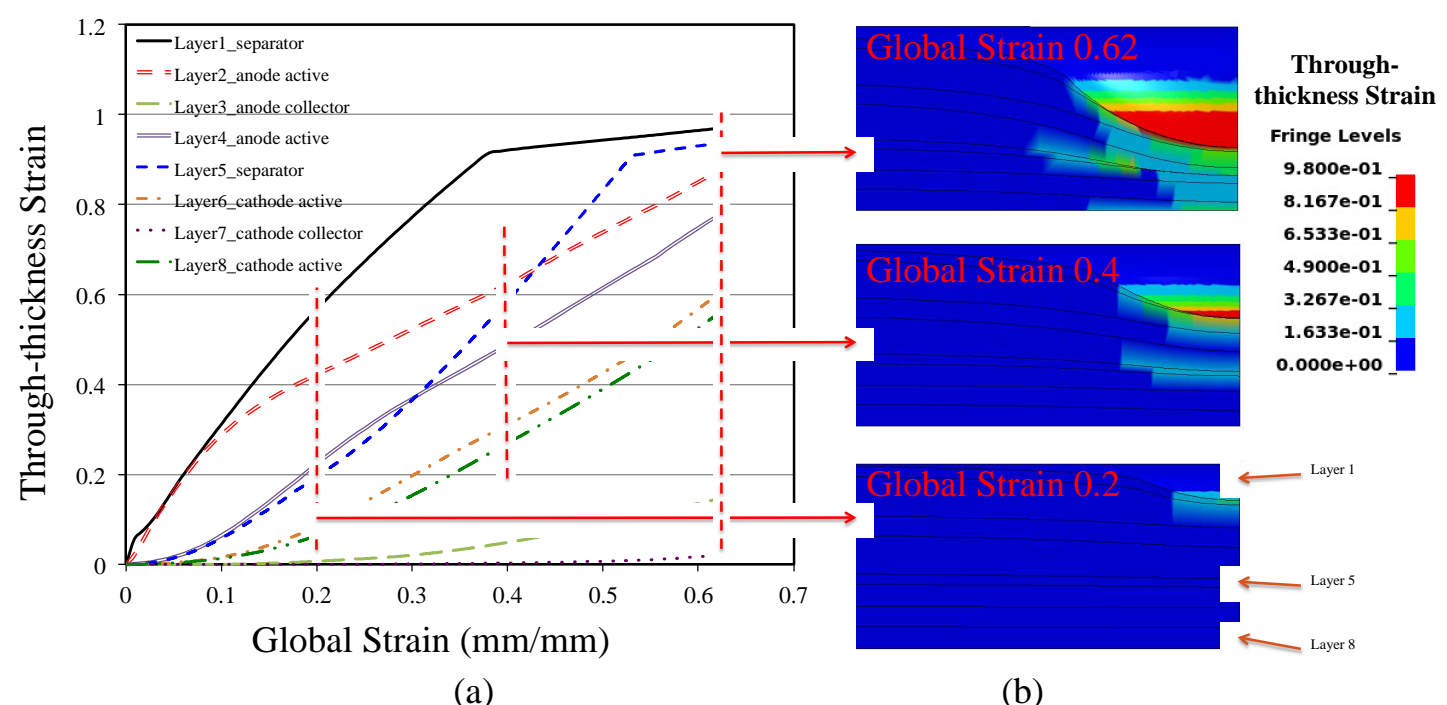

Figure 5 


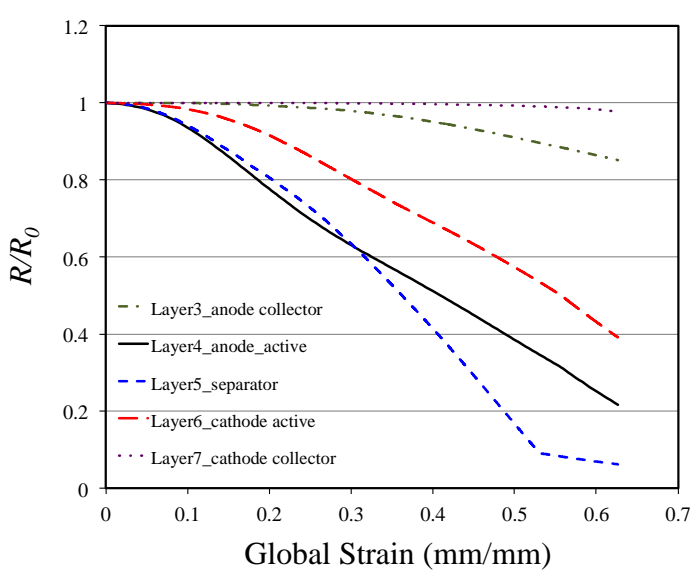

(a)

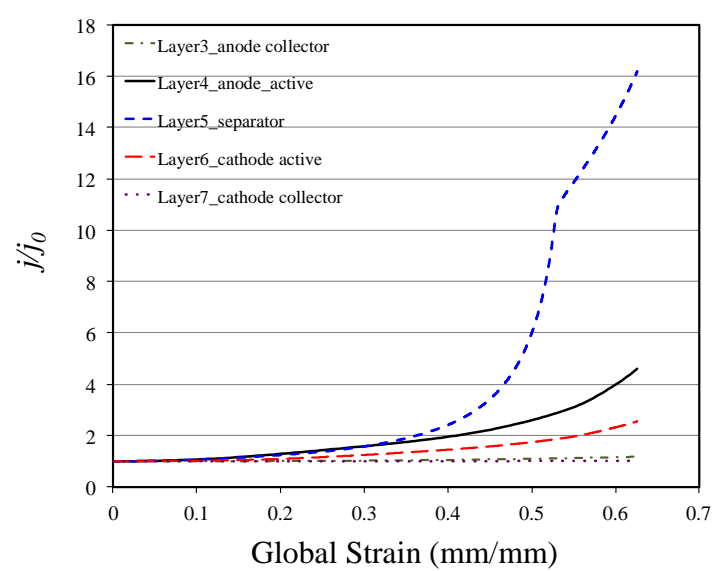

(b)

Figure 6 


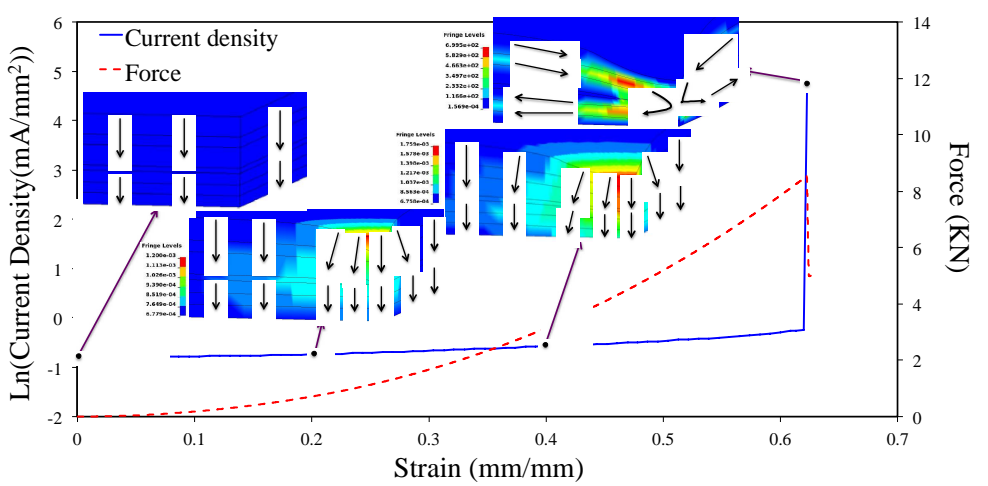

(a)

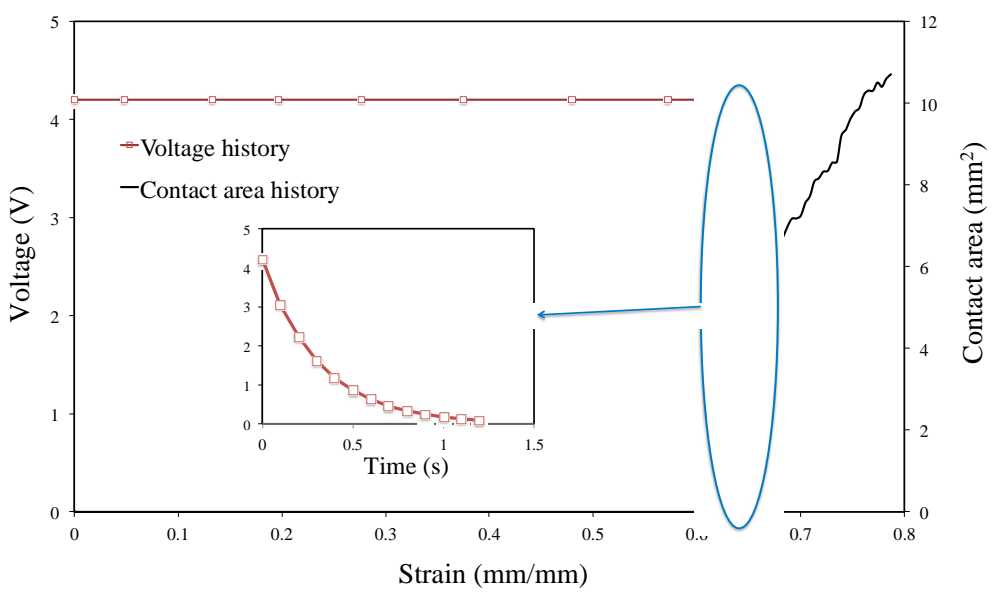

(b)

Figure 7 


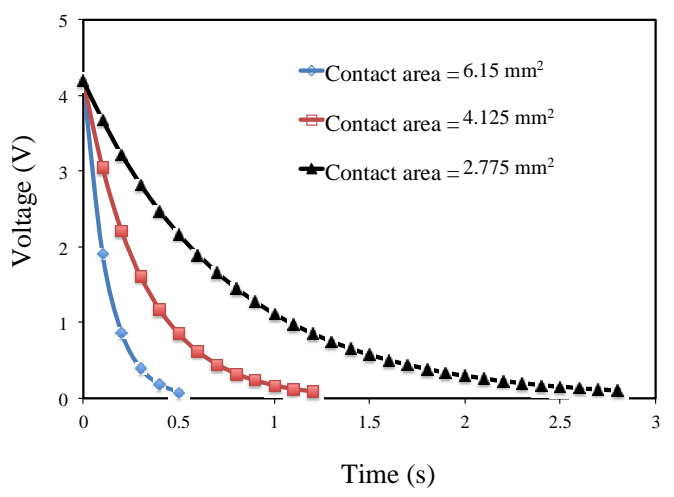

(a)

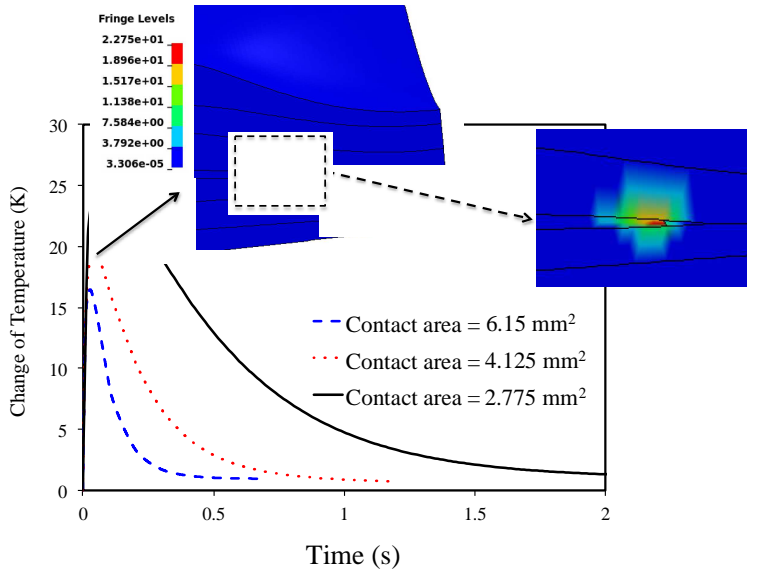

(b)

Figure 8 